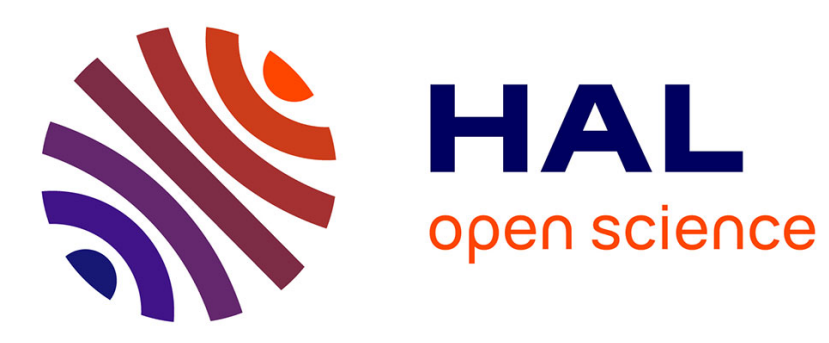

\title{
Relaxed model for the hysteresis in micromagnetism
}

Gilles Carbou, Messoud Efendiev, Pierre Fabrie

\section{To cite this version:}

Gilles Carbou, Messoud Efendiev, Pierre Fabrie. Relaxed model for the hysteresis in micromagnetism. Proceedings of the Royal Society of Edinburgh: Section A, Mathematics, 2008, à paraître. hal00293625

\section{HAL Id: hal-00293625 \\ https://hal.science/hal-00293625}

Submitted on 7 Jul 2008

HAL is a multi-disciplinary open access archive for the deposit and dissemination of scientific research documents, whether they are published or not. The documents may come from teaching and research institutions in France or abroad, or from public or private research centers.
L'archive ouverte pluridisciplinaire $\mathbf{H A L}$, est destinée au dépôt et à la diffusion de documents scientifiques de niveau recherche, publiés ou non, émanant des établissements d'enseignement et de recherche français ou étrangers, des laboratoires publics ou privés. 


\section{Relaxed model for the hysteresis in micromagnetism}

\section{G. Carbou $^{1}$, M. Effendiev ${ }^{2}$ and P. Fabrie ${ }^{1}$}

${ }^{1}$ Institut de Mathématiques de Bordeaux, UMR 5251, Université de Bordeaux, 351 cours de la Libération, 33405 Talence cedex, France.

${ }^{2}$ Universität Stuttgart, Universitätsbereich Vaihingen, Pfaffenwaldring 57, 70550 Stuttgart, Germany.

Abstract : in this paper we study a model of ferromagnetic material with hysteresis effects. The magnetic moment behaviour is described by the non-linear Landau-Lifschitz equation with an additional term modelling the hysteresis. This term takes the form of a maximal monotone operator acting on the time derivative of the magnetic moment. In our model, it is approximated via a relaxing heat equation. For this relaxed model we prove local existence of regular solutions.

\section{Introduction}

The hysteresis properties of the ferromagnetic materials are a very wide domain in physics (see E. Della Torre [4]). The Preisach model describing the magnetic hysteresis is obtained by a phenomenological approach (see [9]). It is explained from the mathematical point of view by A. Visintin in [12]. Other models for rate-independent evolution in ferromagnetic materials are given in [10]. The same kind of models are used for other applications in [7] and [8].

With a physical approach, W. F. Brown developed in [2] the micromagnetism theory. The model described by Landau and Lifschitz in [6] is the following. The magnetic moment $m$ is a unitary vector field linking the magnetic field and the magnetic induction by the relation $B=H+m$. The variations of $m$ are described by the Landau-Lifschitz equation:

$$
\frac{\partial m}{\partial t}=-m \wedge \mathcal{H}_{e f f}-m \wedge\left(m \wedge \mathcal{H}_{e f f}\right),
$$

where the effective field is given by $\mathcal{H}_{\text {eff }}=\Delta m+h_{d}(m)+H_{a}+\Psi(m)$, and the demagnetizing field $h_{d}(m)$ is solution of the magnetostatic equations

$$
\operatorname{div}\left(h_{d}(m)+m\right)=0 \text { and } \operatorname{curl} h_{d}(m)=0,
$$

where $H_{a}$ is an applied magnetic field and where $\Psi(m)$ is an anisotropic term.

Micromagnetic modeling and Preisach modeling are two complementary approaches but the links between these two models are not clear. Using a two time-scales asymptotic method, J. Starynkévitch [11] gives a first answer to bring to the fore the hysteresis in Landau-Lifschitz model. We study here a model due to M. Effendiev. The hysteresis effect in Landau-Lifschitz equation is reinforced by an additional term in the effective field. This term is described with the maximal monotone operator $\beta$ defined as follows

$$
\beta(\xi)=\left\{\begin{array}{l}
\frac{\xi}{|\xi|} \text { if } \xi \neq 0, \\
B(0,1) \text { if } \xi=0 .
\end{array}\right.
$$


In this model the effective field is given by:

$$
\mathcal{H}_{e f f}=\Delta m+h_{d}(m)+H_{a}+\Psi(m)-\beta\left(\frac{\partial m}{\partial t}\right) .
$$

The existence of regular solutions for the system (1.1)-(1.4) is open. We propose here a relaxation model for this system:

$$
\left\{\begin{array}{l}
\frac{\partial m}{\partial t}=m \wedge\left(\Delta m+h_{d}(m)+H_{a}+\Psi(m)-v\right)-m \wedge\left(m \wedge\left(\Delta m+h_{d}(m)+H_{a}+\Psi(m)-v\right)\right) \\
\frac{\partial v}{\partial t}=\Delta v+\frac{1}{\varepsilon}\left(\beta\left(\frac{\partial m}{\partial t}\right)-v\right) \\
\frac{\partial m}{\partial n}=\frac{\partial v}{\partial n}=0 \text { on } \partial \Omega \\
m(t=0)=m_{0} \text { and } v(t=0)=v_{0} \text { on } \Omega .
\end{array}\right.
$$

We prove an existence result of strong solutions for this relaxed system for $\varepsilon>0$ fixed.

We assume that the initial data satisfies the following conditions:

$$
\left\{\begin{array}{l}
m_{0} \in H^{2}(\Omega) \text { and } v_{0} \in H^{1}(\Omega), \\
\left|m_{0}\right|=1 \text { on } \Omega \\
\frac{\partial m_{0}}{\partial n}=0 \text { on } \partial \Omega
\end{array}\right.
$$

For regular solutions, the equation (1.5) with initial data satisfying (1.6) is equivalent to the following system (see [3]):

$$
\left\{\begin{aligned}
& \frac{\partial m}{\partial t}-\Delta m=m|\nabla m|^{2}+m \wedge \Delta m+m \wedge\left(h_{d}(m)+H_{a}+\Psi(m)-v\right) \\
&-m \wedge\left(m \wedge\left(h_{d}(m)+H_{a}+\Psi(m)-v\right)\right) \\
& \frac{\partial v}{\partial t}-\Delta v=\frac{1}{\varepsilon}\left(\beta\left(\frac{\partial m}{\partial t}\right)-v\right) \\
& \frac{\partial m}{\partial n}=\frac{\partial v}{\partial n}=0 \text { on } \partial \Omega, \\
& m(t=0)=m_{0} \text { and } v(t=0)=v_{0} \text { on } \Omega .
\end{aligned}\right.
$$

Indeed if $(m, v)$ is a regular solution of (1.5) then the punctual norm of $m$ is preserved and so $|m|=1$. Then we have $\Delta|m|^{2}=0=m \cdot \Delta m+|\nabla m|^{2}$.

So $m \wedge(m \wedge \Delta m)=(m \cdot \Delta m) m-|m|^{2} \Delta m=-\Delta m-m|\nabla m|^{2}$.

In addition if $(m, v)$ is a regular solution of (1.7) then $|m|^{2}$ satisfies a parabolic equation which unique solution is $|m|^{2} \equiv 1$. Then the previous computation remains valid and $(m, v)$ satisfies $(1.5)$.

Our main result is the following theorem: 
Theorem 1.1 We fix $\varepsilon>0$. Let $\left(m_{0}, v_{0}\right)$ satisfying (1.6). Then there exists $T^{*}>0$, there exists $(m, v)$ solution of (1.7) such that for all $T<T^{*}$,

$$
m \in \mathcal{C}^{0}\left(0, T ; H^{2}(\Omega)\right) \cap L^{2}\left(0, T ; H^{3}(\Omega)\right), \quad v \in \mathcal{C}^{0}\left(0, T ; H^{1}(\Omega)\right) \cap L^{2}\left(0, T ; H^{2}(\Omega)\right) .
$$

In the following section we recall technical lemmas about equivalent norms in the $H^{p}$ spaces, about the demagnetizing field $h_{d}$ and about the maximal monotone operator $\beta$.

In the last section we prove Theorem 1.1.

\section{Technical lemmas}

\section{$2.1 \quad$ Estimates tolls}

The results of this subsection are proved in [3].

Lemma 2.1 Let $\Omega$ be a bounded regular open set. There exists a constant $C$ such that for all $u \in H^{2}(\Omega)$ satisfying $\frac{\partial u}{\partial \nu}=0$ on $\partial \Omega$, we have

$$
\begin{gathered}
\|u\|_{H^{2}(\Omega)} \leq C\left(\|u\|_{L^{2}(\Omega)}^{2}+\|\Delta u\|_{L^{2}(\Omega)}^{2}\right)^{\frac{1}{2}}, \\
\|\nabla u\|_{H^{1}(\Omega)} \leq C\left(\|\nabla u\|_{L^{2}(\Omega)}^{2}+\|\Delta u\|_{L^{2}(\Omega)}^{2}\right)^{\frac{1}{2}},
\end{gathered}
$$

and for $u \in H^{3}(\Omega)$ such that $\frac{\partial u}{\partial \nu}=0$ on $\partial \Omega$,

$$
\|\nabla u\|_{H^{2}(\Omega)} \leq C\left(\|\nabla u\|_{L^{2}(\Omega)}^{2}+\|\Delta u\|_{L^{2}(\Omega)}^{2}+\|\nabla \Delta u\|_{L^{2}(\Omega)}^{2}\right)^{\frac{1}{2}} .
$$

Using Lemma 2.1 and the classical interpolation inequality, we rewrite Sobolev and GagliardoNirenberg inequalities on the following form:

Lemma 2.2 Let $\Omega$ be a regular bounded domain of $\mathbb{R}^{3}$. There exists a constant $C$ such that for all $u \in H^{2}(\Omega)$ such that $\frac{\partial u}{\partial n}=0$ on $\partial \Omega$,

$$
\begin{gathered}
\|u\|_{L^{\infty}(\Omega)} \leq C\left(\|u\|_{L^{2}(\Omega)}^{2}+\|\Delta u\|_{L^{2}(\Omega)}^{2}\right)^{\frac{1}{2}}, \\
\|\nabla u\|_{L^{6}(\Omega)} \leq C\left(\|u\|_{L^{2}(\Omega)}^{2}+\|\Delta u\|_{L^{2}(\Omega)}^{2}\right)^{\frac{1}{2}}, \\
\|\nabla u\|_{L^{4}(\Omega)}^{2} \leq C\|u\|_{L^{\infty}(\Omega)}\left(\|u\|_{L^{2}}^{2}+\|\Delta u\|_{L^{2}(\Omega)}^{2}\right)^{\frac{1}{2}},
\end{gathered}
$$

and for all $u \in H^{3}(\Omega)$ such that $\frac{\partial u}{\partial \nu}=0$ on $\partial \Omega$,

$$
\left\|D^{2} u\right\|_{L^{3}(\Omega)} \leq C\left(\left(\|u\|_{L^{2}(\Omega)}^{2}+\|\Delta u\|_{L^{2}(\Omega)}^{2}\right)^{\frac{1}{2}}+\left(\|u\|_{L^{2}(\Omega)}^{2}+\|\Delta u\|_{L^{2}(\Omega)}^{2}\right)^{\frac{1}{4}}\|\nabla \Delta u\|_{L^{2}(\Omega)}^{\frac{1}{2}}\right)
$$




\subsection{Demagnetizing field}

We consider the operator $m \mapsto h_{d}(m)$ defined by (1.2). It satisfies

$$
\begin{cases}h_{d}(m) \in L^{2}\left(\mathbb{R}^{3}\right), & \\ \operatorname{curl} h_{d}(m)=0 & \text { in } \mathbb{R}^{3}, \\ \operatorname{div}\left(h_{d}(m)+\bar{m}\right)=0 & \text { in } \mathbb{R}^{3},\end{cases}
$$

where $\bar{m}$ is the extension of $m$ by zero outside $\bar{\Omega}$.

We observe that $m \mapsto-h_{d}(m)$ is the orthogonal projection of $\bar{m}$ on the vector fields of gradients in $L^{2}\left(\mathbb{R}^{3}\right)$. We prove in [3] the following estimates concerning the operator $h_{d}$ :

Lemma 2.3 Let $p \in] 1,+\infty\left[\right.$. Then, if $m$ belongs to $W^{1, p}(\Omega)$ (resp. $W^{2 . p}(\Omega)$ ), the restriction of $h_{d}(m)$ to $\Omega$ belongs to $W^{1, p}(\Omega)$ (resp. $W^{2 \cdot p}(\Omega)$ ) and there exists a constant $C$ such that

$$
\begin{gathered}
\left\|h_{d}(m)\right\|_{L^{p}(\Omega)} \leq c\|m\|_{L^{p}(\Omega)}, \quad 1<p<+\infty . \\
\left\|h_{d}(m)\right\|_{W^{1, p}(\Omega)} \leq C\|m\|_{W^{1, p}(\Omega)},
\end{gathered}
$$

and

$$
\left\|h_{d}(m)\right\|_{W^{2, p}(\Omega)} \leq C\|m\|_{W^{2, p}(\Omega)} .
$$

\subsection{Maximal monotone operators tools}

We remark that $\beta$ is a maximal monotone operator. We recall usefull results proved in [1]. The first proposition is about the approximation of $\beta$ by a continuous operator:

Proposition 2.1 For $\lambda>0$ we define $\beta_{\lambda}$ by

$$
\beta_{\lambda}(\xi)=\left\{\begin{array}{l}
\frac{\xi}{|\xi|} \text { for }|\xi| \geq \lambda \\
\frac{\xi}{\lambda} \text { for }|\xi| \leq \lambda .
\end{array}\right.
$$

Then if $\xi_{\lambda}$ tends to $\xi$ uniformly on $[0, T] \times \Omega$ then extracting a subsequence, $\beta_{\lambda}\left(\xi_{\lambda}\right)$ tends to $\beta(\xi)$ in $L^{\infty}$ weak *.

In order to take the limit in a maximal monotone operator, we have the following lemma:

Proposition 2.2 If $A$ is a maximal monotone operator, if $y_{n} \in A\left(x_{n}\right)$, if $x_{n} \rightarrow x$ and $y_{n} \rightarrow y$, if lim sup $<x_{n}\left|y_{n}>\leq<x\right| y>$, then $y \in A(x)$ and $<x_{n}\left|y_{n}>\longrightarrow<x\right| y>$.

\section{$3 \quad$ Proof of Theorem 1.1}

\subsection{First Step : Galerkin Approximation}

We denote by $V_{n}$ the finite dimension space built on the $n$ first eigen-functions of $-\Delta+I d$ with domain $D(A)=\left\{u \in H^{2}(\Omega), \frac{\partial u}{\partial \nu}=0\right.$ on $\left.\partial \Omega\right\}$, and by $\mathbf{P}_{n}$ the orthogonal projection from $L^{2}(\Omega)$ on $V_{n}$. 
We first solve the Galerkine approximation for system (1.7). We fix $n$ and we want to build $\left(m_{n}, v_{n}\right)$ the solution of the following approximate problem:

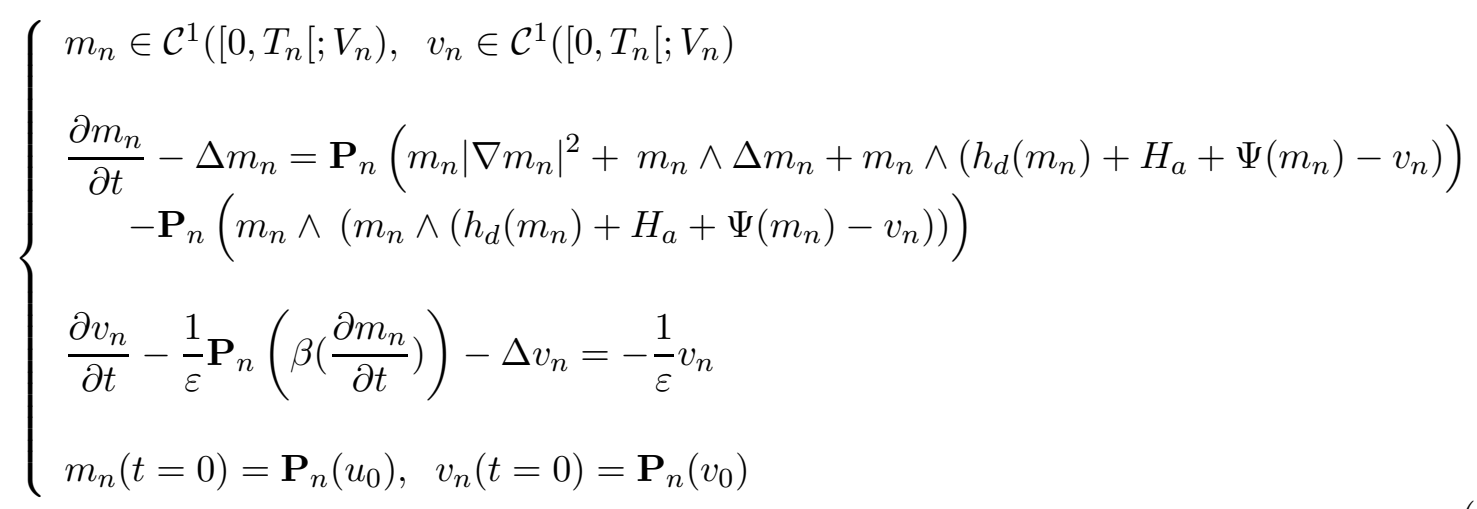

In order to solve this problem and to take into account the specificity of the maximal monotone operator $\beta$, we consider the approximation $\beta_{\lambda}$ of $\beta$, described in the previous section, and we solve the following equation:

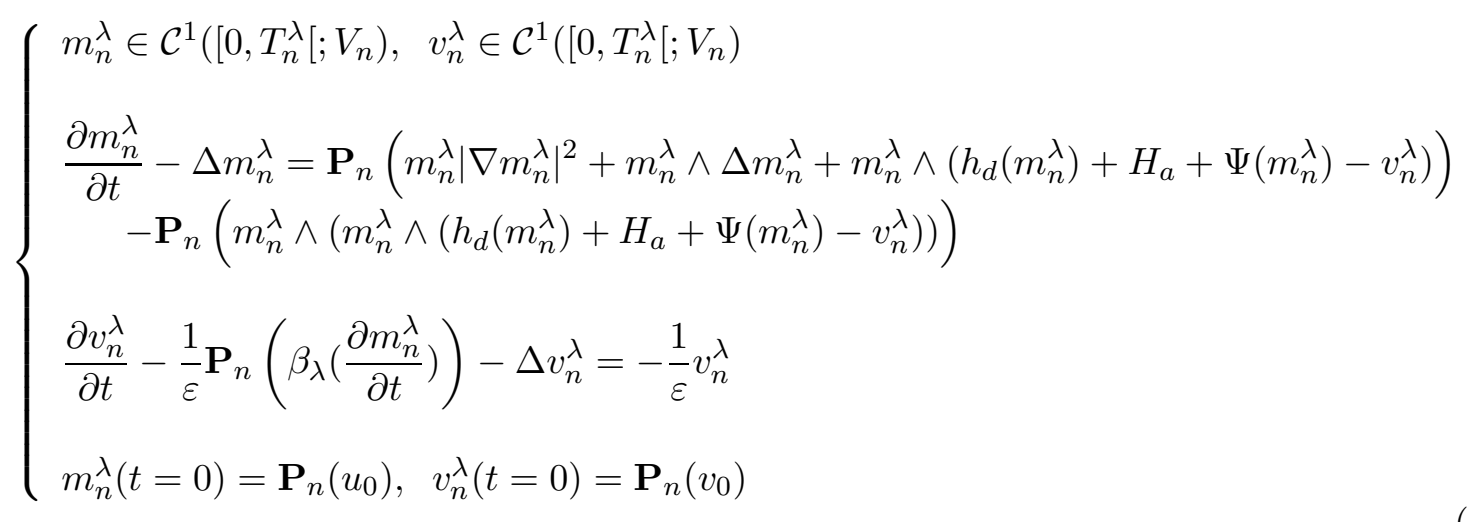

This equation can be written on the following form:

$$
\left\{\begin{array}{l}
m_{n}^{\lambda} \in \mathcal{C}^{1}\left(\left[0, T_{n}^{\lambda}\left[; V_{n}\right), \quad v_{n}^{\lambda} \in \mathcal{C}^{1}\left(\left[0, T_{n}^{\lambda}\left[; V_{n}\right)\right.\right.\right.\right. \\
\frac{\partial m_{n}^{\lambda}}{\partial t}=F_{n}\left(m_{n}^{\lambda}, v_{n}^{\lambda}\right) \\
\frac{\partial v_{n}^{\lambda}}{\partial t}-\frac{1}{\varepsilon} \mathbf{P}_{n}\left(\beta_{\lambda}\left(\frac{\partial m_{n}^{\lambda}}{\partial t}\right)\right)=G\left(v_{n}^{\lambda}\right) \\
m_{n}^{\lambda}(t=0)=\mathbf{P}_{n}\left(u_{0}\right), \quad v_{n}^{\lambda}(t=0)=\mathbf{P}_{n}\left(v_{0}\right)
\end{array}\right.
$$

where $F_{n}: V_{n} \times V_{n} \longrightarrow V_{n}$ and $G: V_{n} \longrightarrow V_{n}$ are smooth. Since we can replace the second equation by

$$
\frac{\partial v_{n}^{\lambda}}{\partial t}=\frac{1}{\varepsilon} \mathbf{P}_{n}\left(\beta_{\lambda}\left(F_{n}\left(m_{n}^{\lambda}, v_{n}^{\lambda}\right)\right)\right)+G\left(v_{n}^{\lambda}\right)
$$

for a fixed $\lambda$ we can apply the Cauchy-Lisfchitz theorem on the finite dimensional space $V_{n} \times V_{n}$ : there exists a unique solution for equation (3.3) defined on the maximal interval $\left[0, T_{n}^{\lambda}[\right.$.

Since $\left\|\beta_{\lambda}(\xi)\right\|_{L^{\infty}(\Omega)} \leq 1$, there exists $K$ depending only on $n$ such that for all $w \in V_{n}$ we have:

$$
\left\|\mathbf{P}_{n}\left(\beta_{\lambda}(w)\right)\right\|_{V_{n}} \leq K .
$$


Since $G$ is linear, we can obtain from (3.4) that there exists a constant $C$ depending on $n$ such that for all $t \leq T_{n}^{\lambda}$ we have:

$$
\left\|v_{n}^{\lambda}\right\|_{V_{n}} \leq C e^{C t} .
$$

Now, there exists a constant $K$ depending on $n$ such that for $(u, v) \in V_{n} \times V_{n}$ we have

$$
\left\|F_{n}(u, v)\right\|_{V_{n}} \leq K_{n}^{\prime}\left(\|u\|_{V_{n}}^{4}+\|v\|_{V_{n}}^{2}\right) .
$$

By comparison lemma we then obtain that there exists a time $T^{n}>0$ such that for all $\lambda>0$, $T_{n}^{\lambda} \geq T^{n}$, and there exists a constant $K_{n}$ such that for all $\lambda$,

$$
\left\|m_{n}^{\lambda}\right\|_{L^{\infty}\left(0, T^{n}\right)}+\left\|v_{n}^{\lambda}\right\|_{L^{\infty}\left(0, T^{n}\right)} \leq K_{n} .
$$

Using (3.5) in (3.3), we obtain a bound for $\frac{\partial m_{n}^{\lambda}}{\partial t}$ and $\frac{\partial v_{n}^{\lambda}}{\partial t}$, and derivating the first equation of (3.3) with respect to $t$, we obtain a bound of $\frac{\partial^{2} u_{n}^{\lambda}}{\partial t^{2}}$. Thus there exists a constant $K$ such that for all $\lambda$,

$$
\left\|m_{n}^{\lambda}\right\|_{L^{\infty}\left(0, T^{n}\right)}+\left\|\frac{\partial m_{n}^{\lambda}}{\partial t}\right\|_{L^{\infty}\left(0, T^{n}\right)}+\left\|\frac{\partial^{2} m_{n}^{\lambda}}{\partial t^{2}}\right\|_{L^{\infty}\left(0, T^{n}\right)}+\left\|v_{n}^{\lambda}\right\|_{L^{\infty}\left(0, T^{n}\right)}+\left\|\frac{\partial v_{n}^{\lambda}}{\partial t}\right\|_{L^{\infty}\left(0, T^{n}\right)} \leq K_{n} .
$$

For a fixed $n$ we take the limit when $\lambda$ tends to zero. From (3.6) we obtain that there exists $m_{n}$ and $v_{n}$ such that

$$
\begin{aligned}
& m_{n}^{\lambda} \longrightarrow m_{n} \text { in } L^{\infty}\left(0, T_{n}\right) \\
& \frac{\partial m_{n}^{\lambda}}{\partial t} \longrightarrow \frac{\partial m_{n}}{\partial t} \text { in } L^{\infty}\left(0, T_{n}\right) \\
& v_{n}^{\lambda} \longrightarrow v_{n} \text { in } L^{\infty}\left(0, T_{n}\right)
\end{aligned}
$$

In addition using Proposition 2.1, we have that $\beta^{\lambda}\left(\frac{\partial m_{n}^{\lambda}}{\partial t}\right)$ tends to $w_{n}$ and $w_{n} \in \beta\left(\frac{\partial m_{n}}{\partial t}\right)$. Furthermore we can take the limit when $\lambda$ tends to zero in Equation (3.2) and we obtain that there exist $T_{n}>0, m_{n} \in \mathcal{C}^{1}\left(\left[0, T_{n}\left[; V_{n}\right)\right.\right.$ and $v_{n} \in \mathcal{C}^{1}\left(\left[0, T_{n}\left[; V_{n}\right)\right.\right.$ satisfying $(3.1)$.

\subsection{Estimates for $m_{n}$ and $v_{n}$}

Taking the inner product in $L^{2}(\Omega)$ of the first equation in (3.1) with $m_{n}$ we obtain that

$$
\frac{1}{2} \frac{d}{d t}\left(\left\|m_{n}\right\|_{L^{2}(\Omega)}^{2}\right)+\left\|\nabla m_{n}\right\|_{L^{2}(\Omega)}^{2} \leq\left\|m_{n}\right\|_{L^{\infty}(\Omega)}^{2}\left\|\nabla m_{n}\right\|_{L^{2}(\Omega)}^{2} .
$$

Taking the inner product in $L^{2}(\Omega)$ of the second equation in (3.1) with $v_{n}$ we obtain that

$$
\frac{1}{2} \frac{d}{d t}\left(\left\|v_{n}\right\|_{L^{2}(\Omega)}^{2}\right)+\left\|\nabla v_{n}\right\|_{L^{2}(\Omega)}^{2} \leq K\left(1+\left\|v_{n}\right\|_{L^{2}(\Omega)}^{2}\right)
$$

since $\left\|\beta\left(\frac{\partial m_{n}}{\partial t}\right)\right\|_{L^{2}(\Omega)} \leq K$. 
We take the inner product in $L^{2}(\Omega)$ of the second equation in (3.1) with $\Delta v_{n}$. Integrating by part the right hand side, and absorbing $\left\|\Delta v_{n}\right\|_{L^{2}(\Omega)}$ using that $\left\|\beta\left(\frac{\partial m_{n}}{\partial t}\right)\right\|_{L^{2}(\Omega)} \leq K$, we obtain that

$$
\frac{1}{2} \frac{d}{d t}\left(\left\|\nabla v_{n}\right\|_{L^{2}(\Omega)}^{2}\right)+\left\|\Delta v_{n}\right\|_{L^{2}(\Omega)}^{2} \leq K\left(1+\left\|\nabla v_{n}\right\|_{L^{2}(\Omega)}^{2}\right)
$$

We take the inner product in $L^{2}(\Omega)$ of the second equation in (3.1) with $\Delta^{2} m_{n}$. We obtain that:

$$
\frac{1}{2} \frac{d}{d t}\left(\left\|\Delta m_{n}(t)\right\|_{L^{2}(\Omega)}^{2}\right)+\left\|\nabla \Delta m_{n}(t)\right\|_{L^{2}(\Omega)}^{2}=I_{1}+I_{2}+I_{3}+I_{4}+I_{5}
$$

with

$$
\begin{aligned}
& I_{1}=\int_{\Omega} \nabla\left(\left|\nabla m_{n}\right|^{2} m_{n}\right) \nabla \Delta m_{n} d x, \\
& I_{2}=\int_{\Omega} \nabla\left(m_{n} \wedge \Delta m_{n}\right) \nabla \Delta m_{n} d x \\
& I_{3}=\int_{\Omega} \nabla\left(m_{n} \wedge h_{d}\left(m_{n}\right)-m_{n} \wedge\left(m_{n} \wedge h_{d}\left(m_{n}\right)\right)\right) \nabla \Delta m_{n} d x, \\
& I_{4}=\int_{\Omega} \nabla\left(m_{n} \wedge\left(H_{a}+\Psi\left(m_{n}\right)\right)-m_{n} \wedge\left(m_{n} \wedge\left(H_{a}+\Psi\left(m_{n}\right)\right)\right)\right) \nabla \Delta m_{n} d x, \\
& I_{5}=\int_{\Omega} \nabla\left(m_{n} \wedge v_{n}-m_{n} \wedge\left(m_{n} \wedge v_{n}\right)\right) \cdot \nabla \Delta m_{n} .
\end{aligned}
$$

We bound separately each term.

- Estimate on $I_{1}$

$$
\begin{aligned}
\left|I_{1}\right| & \leq \int_{\Omega}\left|\nabla m_{n}\right|^{3}\left|\nabla \Delta m_{n}\right| d x+\int_{\Omega}\left|D^{2} m_{n}\left\|\nabla m_{n}\right\| m_{n}\right|\left|\nabla \Delta m_{n}\right| d x, \\
& \leq\left\|\nabla m_{n}\right\|_{L^{6}(\Omega)}^{3}\left\|\nabla \Delta m_{n}\right\|_{L^{2}(\Omega)}+\left\|m_{n}\right\|_{L^{\infty}(\Omega)}\left\|D^{2} m_{n}\right\|_{L^{3}(\Omega)}\left\|\nabla m_{n}\right\|_{L^{6}(\Omega)}\left\|\nabla \Delta m_{n}\right\|_{L^{2}(\Omega)}
\end{aligned}
$$

hence using the Sobolev embeding and Lemmas 2.1 and 2.2 we obtain that there exists a constant $K$ independant of $n$ such that

$$
\begin{aligned}
\left|I_{1}\right| & \leq K\left(\left\|m_{n}\right\|_{L^{2}(\Omega)}^{2}+\left\|\Delta m_{n}\right\|_{L^{2}(\Omega)}^{2}\right)^{\frac{3}{2}}\left\|\nabla \Delta m_{n}\right\|_{L^{2}(\Omega)} \\
& +K\left(\left\|m_{n}\right\|_{L^{2}(\Omega)}^{2}+\left\|\Delta m_{n}\right\|_{L^{2}(\Omega)}^{2}\right)^{\frac{5}{4}}\left\|\nabla \Delta m_{n}\right\|_{L^{2}(\Omega)}^{\frac{3}{2}}
\end{aligned}
$$

- Estimate on $I_{2}$

By Sobolev embeddings and interpolation, we obtain that

$$
\begin{aligned}
\left|I_{2}\right| \leq & \left\|\nabla m_{n}\right\|_{L^{6}(\Omega)}\left\|\Delta m_{n}\right\|_{L^{3}(\Omega)}\|\| \nabla \Delta m_{n} \|_{L^{2}(\Omega)} \\
\leq & K\left(\left\|m_{n}\right\|_{L^{2}(\Omega)}^{2}+\left\|\Delta m_{n}\right\|_{L^{2}(\Omega)}^{2}\right)\left\|\nabla \Delta m_{n}\right\|_{L^{2}(\Omega)}+ \\
& K\left(\left\|m_{n}\right\|_{L^{2}(\Omega)}^{2}+\left\|\Delta m_{n}\right\|_{L^{2}(\Omega)}^{2}\right)^{\frac{3}{4}}\left\|\nabla \Delta m_{n}\right\|_{L^{2}(\Omega)}^{\frac{3}{2}} .
\end{aligned}
$$


- Estimate on $I_{3}$

We have

$\left|I_{3}\right| \leq\left(1+\left\|m_{n}\right\|_{L^{\infty}(\Omega)}\right)\left(\left\|\nabla m_{n}\right\|_{L^{2}(\Omega)}\left\|h_{d}\left(m_{n}\right)\right\|_{L^{2}(\Omega)}+\left\|m_{n}\right\|_{L^{2}(\Omega)}\left\|\nabla h_{d}\left(m_{n}\right)\right\|_{L^{2}(\Omega)}\right)\left\|\nabla \Delta m_{n}\right\|_{L^{2}(\Omega)}$,

and using Lemmas 2.1, 2.2 and 2.3 we obtain that there exists a constant $K$ such that

$$
\left|I_{3}\right| \leq K\left(1+\left(\left\|m_{n}\right\|_{L^{2}(\Omega)}^{2}+\left\|\Delta m_{n}\right\|_{L^{2}(\Omega)}^{2}\right)\right)\left(\left\|m_{n}\right\|_{L^{2}(\Omega)}^{2}+\left\|\Delta m_{n}\right\|_{L^{2}(\Omega)}^{2}\right)\left\|\nabla \Delta m_{n}\right\|_{L^{2}(\Omega)} .
$$

- Estimate on $I_{4}$

From the linearity of $\Psi$ we obtain that there exists a constant $K$ such that

$$
\left|I_{4}\right| \leq K\left(1+\left(\left\|m_{n}\right\|_{L^{2}(\Omega)}^{2}+\left\|\Delta m_{n}\right\|_{L^{2}(\Omega)}^{2}\right)\right)\left(\left\|m_{n}\right\|_{L^{2}(\Omega)}^{2}+\left\|\Delta m_{n}\right\|_{L^{2}(\Omega)}^{2}\right)\left\|\nabla \Delta m_{n}\right\|_{L^{2}(\Omega)}
$$

- Estimate on $I_{5}$

We have

$$
\left|I_{5}\right| \leq\left(\left\|m_{n}\right\|_{L^{\infty}(\Omega)}+\left\|m_{n}\right\|_{L^{\infty}(\Omega)}^{2}\right)\left(\left\|v_{n}\right\|_{L^{2}(\Omega)}+\left\|\nabla v_{n}\right\|_{L^{2}(\Omega)}\right)\left\|\nabla \Delta m_{n}\right\|_{L^{2}(\Omega)}
$$

thus there exists a constant $K$ such that

$$
\left|I_{5}\right| \leq K\left(1+\left\|m_{n}\right\|_{L^{\infty}(\Omega)}^{2}\right)\left(\left\|v_{n}\right\|_{L^{2}(\Omega)}+\left\|\nabla v_{n}\right\|_{L^{2}(\Omega)}\right)\left\|\nabla \Delta m_{n}\right\|_{L^{2}(\Omega)} .
$$

Using Gronwall lemma with the estimates (3.9) and (3.8) we obtain that for all $T$ there exists a constant $C(T)$ such that for all $n$

$$
\left\|v_{n}\right\|_{L^{\infty}\left(0, T ; H^{1}(\Omega)\right)}+\left\|v_{n}\right\|_{L^{2}\left(0, T ; H^{2}(\Omega)\right)} \leq C(T)
$$

Thus plugging this estimate on (3.14), adding up estimates (3.7), (3.10), (3.11), (3.12), (3.13) and (3.14), for all $T$ there exists a constant $C(T)$ such that:

$$
\begin{array}{r}
\frac{1}{2} \frac{d}{d t}\left(\left\|m_{n}\right\|_{L^{2}(\Omega)}^{2}+\left\|\Delta m_{n}\right\|_{L^{2}(\Omega)}^{2}\right)+\left\|\nabla \Delta m_{n}\right\|_{L^{2}(\Omega)}^{2} \leq C(T)\left\|\nabla \Delta m_{n}\right\|_{L^{2}(\Omega)} \\
+C(T)\left(\left\|m_{n}\right\|_{L^{2}(\Omega)}^{2}+\left\|\Delta m_{n}\right\|_{L^{2}(\Omega)}^{2}\right)^{2}\left\|\nabla \Delta m_{n}\right\|_{L^{2}(\Omega)} \\
+K\left(\left\|m_{n}\right\|_{L^{2}(\Omega)}^{2}+\left\|\Delta m_{n}\right\|_{L^{2}(\Omega)}^{2}\right)^{\frac{3}{4}}\left\|\nabla \Delta m_{n}\right\|_{L^{2}(\Omega)}^{\frac{3}{2}}
\end{array}
$$

and after absorption of $\left\|\nabla \Delta m_{n}\right\|_{L^{2}(\Omega)}$ in the right hand side term we obtain that for all $T$ there exists a constant $C(T)$ such that

$$
\frac{d}{d t}\left(\left\|m_{n}\right\|_{L^{2}(\Omega)}^{2}+\left\|\Delta m_{n}\right\|_{L^{2}(\Omega)}^{2}\right)+\left\|\nabla \Delta m_{n}\right\|_{L^{2}(\Omega)}^{2} \leq C(T)\left(1+\left(\left\|m_{n}\right\|_{L^{2}(\Omega)}^{2}+\left\|\Delta m_{n}\right\|_{L^{2}(\Omega)}^{2}\right)^{3}\right)
$$

We consider the solution of the following ordinary differential equation :

$$
\left\{\begin{array}{l}
\frac{d}{d t} \xi=C(T)\left(1+\xi^{3}\right) \\
\xi(0)=\left(\left\|m_{0}\right\|_{L^{2}(\Omega)}^{2}+\left\|\Delta m_{0}\right\|_{L^{2}(\Omega)}^{2}\right)
\end{array}\right.
$$


Since for all $n,\left(\left\|\mathbf{P}_{n}\left(m_{0}\right)\right\|_{L^{2}(\Omega)}^{2}+\left\|\Delta \mathbf{P}_{n}\left(m_{0}\right)\right\|_{L^{2}(\Omega)}^{2}\right) \leq\left(\left\|m_{0}\right\|_{L^{2}(\Omega)}^{2}+\left\|\Delta m_{0}\right\|_{L^{2}(\Omega)}^{2}\right)$ we obtain that for all $t$ and for all $n$, we have:

$$
\left(\left\|m_{n}(t)\right\|_{L^{2}(\Omega)}^{2}+\left\|\Delta m_{n}(t)\right\|_{L^{2}(\Omega)}^{2}\right) \leq \xi(t)
$$

and if we denote by $T^{*}$ the lifespan of $\xi$, for all $T<T^{*}$, for all $n$, we have:

$$
\left\|m_{n}\right\|_{L^{\infty}\left(0, T ; H^{2}(\Omega)\right)}+\left\|m_{n}\right\|_{L^{2}\left(0, T ; H^{3}(\Omega)\right)}+\left\|v_{n}\right\|_{L^{\infty}\left(0, T ; H^{1}(\Omega)\right)}+\left\|v_{n}\right\|_{L^{2}\left(0, T ; H^{2}(\Omega)\right)} \leq C(T)
$$

In addition using the equation (3.1) we obtain a bound for $\frac{\partial m_{n}}{\partial t}$ and $\frac{\partial v_{n}}{\partial t}$ :

$$
\left\|\frac{\partial m_{n}}{\partial t}\right\|_{L^{2}\left(0, T ; H^{1}(\Omega)\right)}+\left\|\frac{\partial v_{n}}{\partial t}\right\|_{L^{2}\left(0, T ; L^{2}(\Omega)\right)} \leq C(T) .
$$

\subsection{Limit when $n$ tends to $+\infty$}

From (3.17) we obtain a uniform bound for $m_{n}$ in $L^{\infty}\left(0, T ; H^{2}(\Omega)\right) \cap L^{2}\left(0, T ; H^{3}(\Omega)\right)$ and using the first equation of (3.1) we obtain a uniform bound for $\frac{\partial m_{n}}{\partial t}$ in $L^{2}\left(0, T ; H^{1}(\Omega)\right)$. Thus we can extract a subsequence such that

$$
\left\{\begin{array}{l}
m_{n} \rightarrow m \text { in } L^{\infty}\left(0, T ; H^{2}(\Omega)\right) \text { weak* } \\
m_{n} \rightarrow m \text { in } L^{2}\left(0, T ; H^{3}(\Omega)\right) \text { weak } \\
\frac{\partial m_{n}}{\partial t} \rightarrow \frac{\partial m}{\partial t} \text { in } L^{2}\left(0, T ; H^{1}(\Omega)\right) \text { weak }
\end{array}\right.
$$

In addition, concerning $v_{n}$ we have by (3.17) a uniform bound in $\left.L^{\infty}\left(0, T ; H^{1} \Omega\right)\right) \cap L^{2}\left(0, T ; H^{2}(\Omega)\right)$ and using the second equation of (3.1) we obtain a uniform bound for $\frac{\partial v_{n}}{\partial t}$ in $L^{2}\left(0, T ; L^{2}(\Omega)\right)$. Thus we can extract a subsequence such that

$$
\left\{\begin{array}{l}
v_{n} \rightarrow v \text { in } L^{\infty}\left(0, T ; H^{1}(\Omega)\right) \text { weak* } \\
v_{n} \rightarrow v \text { in } L^{2}\left(0, T ; H^{2}(\Omega)\right) \text { weak } \\
\frac{\partial v_{n}}{\partial t} \rightarrow \frac{\partial v}{\partial t} \text { in } L^{2}\left(0, T ; L^{2}(\Omega)\right) \text { weak }
\end{array}\right.
$$

Since $\mathbf{P}_{n}\left(\beta\left(\frac{\partial m_{n}}{\partial t}\right)\right)$ is uniformly bounded in $L^{\infty}\left(0, T ; L^{2}(\Omega)\right)$ we can assume that

$$
\mathbf{P}_{n}\left(\beta\left(\frac{\partial m_{n}}{\partial t}\right)\right) \rightarrow w \text { in } L^{\infty}\left(0, T ; L^{2}(\Omega)\right) \text { weak* }
$$

Taking the limit in (3.1) we obtain that $m, v$ and $w$ satisfy the following system on the time interval $\left[0, T^{*}[\right.$ :

$$
\left\{\begin{array}{l}
\frac{\partial m}{\partial t}-\Delta m=m|\nabla m|^{2}+m \wedge \Delta m+m \wedge\left(h_{d}(m)+H_{a}+\Psi(m)-v\right) \\
-m \wedge\left(m \wedge\left(h_{d}(m)+H_{a}+\Psi(m)-v\right)\right) \\
\frac{\partial v}{\partial t}-\frac{1}{\varepsilon} w-\Delta v=-\frac{1}{\varepsilon} v \\
m(t=0)=m_{0}, \quad v(t=0)=v_{0}
\end{array}\right.
$$


It remains to prove that $w \in \beta\left(\frac{\partial m}{\partial t}\right)$. We will prove that $\frac{\partial m_{n}}{\partial t}$ tends to $\frac{\partial m}{\partial t}$ strongly in $L^{2}(0, T \times$ $\Omega)$. Then we will apply Proposition 2.2: since $<\frac{\partial m_{n}}{\partial t}\left|\beta\left(\frac{\partial m_{n}}{\partial t}\right)>\longrightarrow<\frac{\partial m}{\partial t}\right| w>$, then $w \in$ $\beta\left(\frac{\partial m}{\partial t}\right)$

We know that $\frac{\partial m_{n}}{\partial t}$ is bounded in $L^{\infty}\left(0, T ; L^{2}(\Omega)\right) \cap L^{2}\left(0, T ; H^{1}(\Omega)\right)$. In order to obtain compactness for $\frac{\partial m_{n}}{\partial t}$, we seek a bound on $\frac{\partial^{2} m_{n}}{\partial t^{2}}$. We have :

$$
\frac{\partial^{2} m_{n}}{\partial t^{2}}=T_{1}+\ldots+T_{7}
$$

where

$$
\begin{aligned}
& T_{1}=\Delta \frac{\partial m_{n}}{\partial t} \\
& T_{2}=\mathbf{P}_{n}\left(m_{n} \wedge \Delta \frac{\partial m_{n}}{\partial t}\right) \\
& T_{3}=\mathbf{P}_{n}\left(\frac{\partial m_{n}}{\partial t}\left|\nabla m_{n}\right|^{2}+\frac{\partial m_{n}}{\partial t} \wedge \Delta m_{n}\right) \\
& T_{4}=\mathbf{P}_{n}\left(2 m_{n} \nabla m_{n} \nabla \frac{\partial m_{n}}{\partial t}\right) \\
& T_{5}=\mathbf{P}_{n}\left(\frac{\partial m_{n}}{\partial t} \wedge\left(H\left(m_{n}\right)-v_{n}\right)-\frac{\partial m_{n}}{\partial t} \wedge\left(m_{n} \wedge\left(H\left(m_{n}\right)-v_{n}\right)\right)-m_{n} \wedge\left(\frac{\partial m_{n}}{\partial t} \wedge\left(H\left(m_{n}\right)-v_{n}\right)\right)\right.
\end{aligned}
$$

where $H\left(m_{n}\right)=h_{d}\left(m_{n}\right)+H_{a}+\Psi\left(m_{n}\right)$

$$
\begin{aligned}
& T_{6}=\mathbf{P}_{n}\left(m_{n} \wedge H\left(\frac{\partial m_{n}}{\partial t}\right)-m_{n} \wedge\left(m_{n} \wedge H\left(\frac{\partial m_{n}}{\partial t}\right)\right)\right. \\
& T_{7}=\mathbf{P}_{n}\left(m_{n} \wedge \frac{\partial v_{n}}{\partial t}-m_{n} \wedge\left(m_{n} \wedge \frac{\partial v_{n}}{\partial t}\right)\right)
\end{aligned}
$$

From (3.17) and (3.18) we estimate each term on the following way:

- $\left\|T_{1}\right\|_{L^{2}\left(0, T ; H^{-1}(\Omega)\right)} \leq K$

- We estimate the $H^{-1}$ norm of $T_{2}$ by duality arguments: for $\varphi \in \mathcal{C}^{1}\left(\left[0, T\left[; H_{0}^{1}(\Omega)\right)\right.\right.$ we have

$$
\begin{aligned}
<\mathbf{P}_{n}\left(m_{n} \wedge \Delta \frac{\partial m_{n}}{\partial t}\right) \mid \varphi>= & -<\Delta \frac{\partial m_{n}}{\partial t} \mid m_{n} \wedge \mathbf{P}_{n}(\varphi)> \\
& =<\nabla \frac{\partial m_{n}}{\partial t}\left|\nabla m_{n} \wedge \mathbf{P}_{n}(\varphi)>+<\nabla \frac{\partial m_{n}}{\partial t}\right| m_{n} \wedge \nabla \mathbf{P}_{n}(\varphi)>
\end{aligned}
$$


We integrate in time and we obtain that

$$
\begin{aligned}
\left|\int_{0}^{T}<T_{2}\right| \varphi>\mid \leq & \left\|\mathbf{P}_{n}\left(\nabla m_{n} \wedge \nabla \frac{\partial m_{n}}{\partial t}\right)\right\|_{L^{\frac{4}{3}}\left(0, T ; H^{-1}(\Omega)\right)}\|\varphi\|_{L^{4}\left(0, T ; H_{0}^{1}(\Omega)\right)} \\
& +\left\|m_{n} \wedge \nabla \frac{\partial m_{n}}{\partial t}\right\|_{L^{2}\left(0, T ; L^{2}(\Omega)\right)}\left\|\nabla \mathbf{P}_{n}(\varphi)\right\|_{L^{2}\left(0, T ; L^{2}(\Omega)\right)} \\
\leq & \left\|\nabla m_{n}\right\|_{L^{4}\left(0, T ; H^{\frac{3}{2}}(\Omega)\right)}\left\|\nabla \frac{\partial m_{n}}{\partial t}\right\|_{L^{2}\left(0, T ; L^{2}(\Omega)\right)}\|\varphi\|_{L^{4}\left(0, T ; H_{0}^{1}(\Omega)\right)} \\
& +\left\|m_{n}\right\|_{L^{\infty}(0, T \times \Omega)}\left\|\nabla \frac{\partial m_{n}}{\partial t}\right\|_{L^{2}\left(0, T ; L^{2}(\Omega)\right)}\|\nabla \varphi\|_{L^{2}\left(0, T ; L^{2}(\Omega)\right)}
\end{aligned}
$$

Hence

$$
\left\|T_{2}\right\|_{L^{\frac{4}{3}}\left(0, T ; H^{-1}(\Omega)\right)} \leq K
$$

- we have

$$
\begin{aligned}
\left\|\frac{\partial m_{n}}{\partial t}\left|\nabla m_{n}\right|^{2}\right\|_{L^{2}\left(0, T ; L^{2}(\Omega)\right)} & \leq\left\|\frac{\partial m_{n}}{\partial t}\right\|_{L^{2}\left(0, T ; L^{6}(\Omega)\right)}\left\|\nabla m_{n}\right\|_{L^{\infty}\left(0, T ; L^{6}(\Omega)\right)} \\
& \leq\left\|\frac{\partial m_{n}}{\partial t}\right\|_{L^{2}\left(0, T ; H^{1}(\Omega)\right)}\left\|\nabla m_{n}\right\|_{L^{\infty}\left(0, T ; H^{1}(\Omega)\right)} .
\end{aligned}
$$

In addition

$$
\left\|\frac{\partial m_{n}}{\partial t} \wedge \Delta m_{n}\right\|_{L^{2}\left(0, T ; L^{\frac{3}{2}}(\Omega)\right)} \leq\left\|\frac{\partial m_{n}}{\partial t}\right\|_{L^{2}\left(0, T ; L^{6}(\Omega)\right)}\left\|m_{n}\right\|_{L^{\infty}\left(0, T ; H^{2}(\Omega)\right)} .
$$

Hence

$$
\left\|T_{3}\right\|_{L^{2}\left(0, T ; H^{-1}(\Omega)\right)} \leq K .
$$

- We have $\left\|\nabla m_{n}\right\|_{L^{4}\left(0, T ; H^{\frac{3}{2}}(\Omega)\right)} \leq K$ by interpolation theorem. Hence, since for all $p<+\infty$, $L^{4}\left(0, T ; H^{\frac{3}{2}}(\Omega)\right) \subset L^{4}\left(0, T ; L^{p}(\Omega)\right)$, we have that for all $\eta>0$,

$$
\begin{aligned}
\left\|T_{4}\right\|_{L^{\frac{4}{3}}\left(0, T ; H^{-1}(\Omega)\right)} & \leq\left\|T_{4}\right\|_{L^{\frac{4}{3}}\left(0, T ; L^{2-\eta}(\Omega)\right)} \\
& \leq\left\|m_{n}\right\|_{L^{\infty}(0, T \times \Omega)}\left\|\nabla m_{n}\right\|_{L^{4}\left(0, T ; H^{\frac{3}{2}}(\Omega)\right)}\left\|\nabla \frac{\partial m_{n}}{\partial t}\right\|_{L^{2}\left(0, T ; L^{2}(\Omega)\right)} \\
& \leq K .
\end{aligned}
$$

- $\frac{\partial m_{n}}{\partial t}$ is bounded in $L^{2}\left(0, T ; L^{6}(\Omega)\right), H\left(m_{n}\right)-v_{n}$ is bounded in $L^{\infty}\left(0, T ; L^{6}(\Omega)\right)$, and $m_{n}$ is bounded in $L^{\infty}(0, T \times \Omega)$. Hence $T_{5}$ is bounded in $L^{2}\left(0, T ; L^{3}(\Omega)\right)$, so there exists a constant $K$ such that

$$
\left\|T_{5}\right\|_{L^{2}\left(0, T ; H^{-1}(\Omega)\right)} \leq K
$$

- $\frac{\partial m_{n}}{\partial t}$ is bounded in $L^{2}\left(0, T ; L^{2}(\Omega)\right)$ hence by property of the operator $h_{d}$ (see Proposition $2.3)$, since $m_{n}$ is bounded in $L^{\infty}(0, T \times \Omega)$,

$$
\left\|T_{6}\right\|_{L^{2}\left(0, T ; L^{2}(\Omega)\right)} \leq K
$$

- $\frac{\partial v_{n}}{\partial t}$ is bounded in $L^{2}\left(0, T ; L^{6}(\Omega)\right)$, therefore since $m_{n}$ is bounded in $L^{\infty}(0, T \times \Omega)$,

$$
\left\|T_{7}\right\|_{L^{2}\left(0, T ; L^{2}(\Omega)\right)} \leq K .
$$


Therefore we obtain that there exists a constant $K$ independant of $n$ such that

$$
\left\|\frac{\partial^{2} m_{n}}{\partial t^{2}}\right\|_{L^{\frac{4}{3}}\left(0, T ; H^{-1}(\Omega)\right)} \leq K .
$$

Now $\frac{\partial m_{n}}{\partial t}$ is bounded in $L^{2}\left(0, T ; H^{1}(\Omega)\right)$. So by Simon's lemma,

$$
\frac{\partial m_{n}}{\partial t} \longrightarrow \frac{\partial m}{\partial t} \text { in } L^{2}\left(0, T ; L^{2}(\Omega)\right) \text { strong. }
$$

We have $w_{n}=\beta\left(\frac{\partial m_{n}}{\partial t}\right) \rightarrow w$ in $L^{2}\left(0, T ; L^{2}(\Omega)\right.$. So

$$
<w_{n}\left|\frac{\partial m_{n}}{\partial t}>\longrightarrow<w\right| \frac{\partial m}{\partial t}>\text {. }
$$

Hence by Proposition 2.2, $w \in \beta\left(\frac{\partial m}{\partial t}\right)$, which concludes the proof of Theorem 1.1.

\section{References}

[1] H. Brezis, Opérateurs maximaux monotones et semi-groupes de contractions dans les espaces de Hilbert, North-Holland Mathematics Studies, No. 5. Notas de Matemática (50). North-Holland Publishing Co., Amsterdam-London; American Elsevier Publishing Co., Inc., New York, 1973.

[2] W. F. Brown, Micromagnetics, Wiley, New York (1963).

[3] Gilles Carbou and Pierre Fabrie, Regular solutions for Landau-Lifschitz equation in a bounded domain, Differential Integral Equations, 14 (2), 213-229 (2001).

[4] E. Della Torre, Problems in physical modeling of magnetic materials, Physica B, 343:1-9, 2004 .

[5] Stéphane Labbé and Pierre-Yves Bertin, Microwave polarisability of ferrite particles with non-uniform magnetization, Journal of Magnetism and Magnetic Materials, 206:93-105, 1999.

[6] L. Landau et E. Lifschitz, Electrodynamique des milieux continues, cours de physique théorique, tome VIII (ed. Mir) Moscou (1969).

[7] A. Mielke, Evolution of rate-independent systems. Evolutionary equations. Vol. II, 461-559, Handb. Differ. Equ., Elsevier/North-Holland, Amsterdam, 2005

[8] A. Mielke, F. Theil, V. I. Levitas. A variational formulation of rate-independent phase transformations using an extremum principle. Arch. Ration. Mech. Anal. 162 (2002), no. $2,137-177$.

[9] F. Preisach, Z. Phys, 94:277, 1935.

[10] T. Roubíček, M. Kružík. Microstructure evolution model in micromagnetics. Z. Angew. Math. Phys. 55 (2004), no. 1.

[11] J. Starynkevitch, Problèmes d'asymptotique en temps en ferromagnétisme, Thèse de l'Université Bordeaux 1, 2006.

[12] A. Visintin, Six Talks on Hysteresis, CRM Proceedings and Lecture Notes, vol 13, 1998. 